\title{
Um modelo de fluxo em arcos para o problema de corte de estoque com modos alternativos de manufatura
}

\author{
Heloisa Vasques da Silva ${ }^{1}$ \\ Faculdade de Engenharia de Bauru, UNESP, Bauru, SP \\ Felipe Kesrouani Lemos ${ }^{2}$ \\ Unoeste, Presidente Prudente, SP \\ Silvio Alexandre de Araujo ${ }^{3}$ \\ Instituto de Biociências, Letras e Ciências Exatas, UNESP, São José do Rio Preto, SP
}

\begin{abstract}
Resumo. O problema de corte de estoque (PCE) apresenta importância reconhecida e crescente, tanto no meio acadêmico, como industrial. Por estar tipicamente imerso em processos produtivos, diversas características operacionais podem ser verificadas em diferentes sistemas de produção. Este artigo propõe-se a contribuir no estudo de uma característica operacional e suas integrações com processos de corte unidimensionais, motivado por um estudo anterior: o problema de minimização de custos com modos alternativos de produção. Uma das aplicações dos modos alternativos de manufatura se encontra quando um produto pode ser produzido com diferentes materiais. O objetivo desse artigo consiste em reformular o problema de corte com modos alternativos de manufatura, proposto na literatura, e analisar o desempenho da abordagem proposta por meio de testes computacionais, cujos resultados indicam que a nova formulação obtém melhores limitantes inferiores e menores tempos de solução comparados com a formulação existente.
\end{abstract}

Palavras-chave. Modelagem matemática, Problema de corte de estoque, Múltiplos modos de manufatura, Fluxo em arcos

\section{Introdução}

O Problema de Corte consiste em determinar a melhor forma de cortar objetos de maneira a produzir um conjunto de itens, ou seja, um conjunto de unidades menores do objeto, de acordo com a necessidade [1]. Este problema aparece em diversos processos industriais de corte, em que os objetos, em geral disponíveis em estoque, correspondem, por exemplo, a barras de aço, bobinas de papel, chapas metálicas ou de madeira, e os itens, com dimensões especificadas, são, em geral, encomendados. Este problema de simples compreensão e grande aplicabilidade prática é NP-difícil [3]. Nas últimas décadas, vários artigos de revisão bibliográfica relacionados ao problema de corte e empacotamento foram publicados: [3], [10] e [1].

O presente artigo tem como tema de estudo um caso particular dos problemas de corte denominado Problema de Corte de Estoque (PCE), em inglês, Cutting Stock Problem, em que se tem em estoque um conjunto de objetos de dimensões fixas, que devem ser cortados para satisfazer a demanda de um conjunto de itens fracamente heterogêneo e, tendo como objetivo, a minimização da quantidade de matéria-prima.

\footnotetext{
${ }^{1}$ heloisa.vasques@unesp.br

2 felipeklemos@gmail.com.

${ }^{3}$ silvio.araujo@unesp.br.
} 
O interesse de pesquisa intenso e crescente neste tipo de problema pode ser explicado por (i) sua aplicabilidade, (ii) diversidade de problemas reais e (iii) complexidade combinatória [8].

Enquanto as primeiras publicações do PCE se concentravam em modelos e métodos para problemas de minimizar perdas sujeito à restrições de atendimento à demanda, atualmente muita atenção tem sido voltada para a integração a outras condições operacionais encontradas em contextos produtivos [12], por exemplo, o problema de corte de estoque integrado ao problema de: dimensionamento de lotes e sequenciamento de produção [7]; e modos alternativos de manufatura [5]. Esse último pode ser encontrado em situações em que o mesmo produto final pode ser produzido com diferentes combinações de itens [5], como, também, quando há um trade-off entre a duração e os requisitos de recursos de um projeto [6].

Esse trabalho se insere no contexto da tendência de integração dos PCE com condições operacionais; e escassez, na literatura, de estudos que integrem o PCE com o problema de modos alternativos de manufatura. Assim, o objetivo deste artigo consiste em: reformular o problema de corte de estoque com modos alternativos de manufatura utilizando outro modelo encontrado na literatura; implementar o modelo e realizar testes computacionais objetivando estudos de desempenho; e comparar os diferentes resultados dos testes.

\section{Definição do problema e modelagem matemática}

Em [5] os autores apresentam a integração entre o problema de corte de estoque unidimensional com modos alternativos de manufatura (PCEMA-1D), com objetivo de minimizar o custo total de matérias-primas para um conjunto de produtos a serem produzidos. A motivação para o estudo foi o processo de corte de barras de ferro para montagem de armaduras estruturais na indústria de artefatos de concreto, onde a existência de diversas espessuras de barras de aço possibilita a combinação de diferentes arranjos para atender a um mesmo requisito de forças cortantes, resultando em múltiplos modos de manufatura. A Figura 1 representa dois possíveis modos de produção que combinam itens com tamanhos e espessuras diferentes, mas resultam no mesmo produto final.

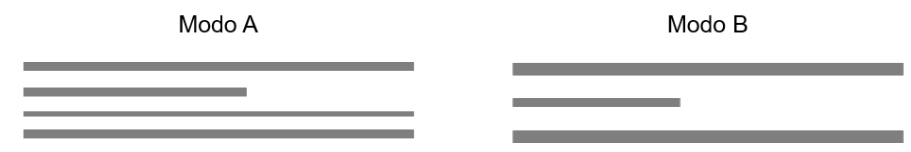

Figura 1: Representação de modos alternativos.

A formulação proposta em [5] faz uso da ideia de padrões de corte, que define a maneira que as peças são cortadas a partir do objeto. Para resolver a relaxação linear deste modelo, os autores utilizaram o método de geração de colunas proposto por [4]. Posteriormente, as colunas geradas foram utilizadas para obter uma solução inteira com o solver CPLEX. Trata-se, então, de um método heurístico, pois a solução inteira é obtida utilizando somente as colunas geradas durante o processo de geração de colunas, ou seja, algumas colunas que possivelmente fariam parte da solução ótima não estão disponíveis. Este tipo de estratégia tem sido bastante utilizada (por exemplo, [7]) e tem obtido bons resultados.

Com o objetivo de reformular o PCEMA-1D de modo que possa ser resolvido de maneira exata por pacotes computacionais, é proposta uma formulação baseada no modelo alternativo para o PCE unidimensional, apresentada por [11] e estudado em [9], que utiliza a ideia de fluxo em arcos.

O problema de encontrar um padrão de corte válido é modelado como um problema de encontrar um caminho em um grafo acíclico direcionado $G=(V, A)$, para o qual $V=0,1, \ldots, L$, é o conjunto de vértices, onde $L$ é o comprimento do objeto, e a distância de um vértice ao outro representa uma unidade de comprimento do objeto. O conjunto de arcos no grafo é definido como $A=\{(a, b)$ : 
$\left.a<b ; b-a=l_{i}, i \in I ; a, b \in V\right\} \cup\left\{(a, a+1): \min \left(l_{i}\right) \leq a, a \in V \backslash\{L\}\right\}$, onde $l_{i}$ é o tamanho do item $i$, sendo que as perdas no objeto geradas do processo de corte são representadas pelos arcos adicionais entre os vértices $(a, a+1)$.

Como o modelo apresenta muitas soluções simétricas, [9] apresentou três critérios de redução para eliminar alguns arcos e reduzir o número de soluções alternativas sem eliminar padrões de cortes válidos do conjunto $A$. O primeiro critério consiste em alocar os itens em ordem decrescente de comprimento, ou seja, um item de comprimento $l_{1}$ só pode ser alocado depois de um item $l_{2}$ se $l_{1} \leq l_{2}$, ou no início do objeto. O segundo critério não permite iniciar um arco com perda. $O$ último critério determina que não são necessários mais arcos do que a demanda do item.

O modelo de fluxo em arcos para o PCEMA-1D é apresentado em (1) - (7) e a seguir estão os conjuntos, parâmetros e variáveis de decisão do modelo.

\section{Conjuntos:}

$J(j \in J, j=1, \ldots, N J)$ : conjunto de ordens de produção de produtos finais a serem fabricados; $K(k \in K, k=1, \ldots, N K)$ : conjunto de espessuras das matérias-primas unidimensionais;

$I(i \in I, i=1, \ldots, N I)$ : conjunto de tamanhos de itens a serem cortados;

$M_{j}\left(m \in M_{j}\right)$ : conjuntos de modos alternativos para o atendimento de uma ordem de produção $j$.

\section{Parâmetros:}

$d_{j}$ : demanda do produto final $j$;

$\theta_{k}$ : custo da matéria-prima de espessura $k$;

$b_{i k m}$ : quantidade de itens de tamanho $l_{i}$ e de espessura $k$ utilizadas no modo de produção $m$.

\section{Variáveis de decisão:}

$F_{k}$ : fluxo total no grafo, isto é, $F_{k}$ é a quantidade de objetos de espessura $k$ utilizados para atender a demanda;

$X_{k a b}$ : número de vezes que a matéria-prima de espessura $k$ é cortada nas posições $a$ e $b$; $Z_{j m}$ : quantidade de produto tipo $j$ produzido no modo de produção $m$.

$$
\min \sum_{k \in K} \theta_{k} F_{k}
$$

s. a.:

$$
\begin{gathered}
-\sum_{(0, b) \in A} X_{k 0 b}=-F_{k}, \forall k \in K \\
\sum_{(a, b) \in A} X_{k a b}-\sum_{(b, c) \in A} X_{k b c}=0, b=1, \ldots, L-1, \forall k \in K \\
\sum_{(a, L) \in A} X_{k a L}=F_{k}, \forall k \in K \\
\sum_{\left(a, a+l_{i}\right) \in A} X_{k a, a+l_{i}} \geq \sum_{j \in J} \sum_{m \in M_{j}} b_{i k m} Z_{j m}, \forall i \in I, k \in K \\
\sum_{m \in M_{j}} Z_{j m} \geq d_{j}, \forall j \in J \\
X_{k a b}, F_{k}, Z_{j m} \in \mathbb{Z}_{+}, \forall(a, b) \in A, k \in K, j \in J, m \in M_{j}
\end{gathered}
$$

A função objetivo (1) minimiza o custo total com matérias-primas. As restrições (2), (3), (4) são de conservação de fluxo, derivadas do modelo de [9]. As restrições (5) garantem o cumprimento da demanda total de cada item $i$ de cada matéria-prima $k$. As restrições (6) fazem com que a soma de todos os produtos $j$ manufaturados em seus possíveis modos $m\left(m \in M_{j}\right)$ seja igual ou superior à demanda deste produto $\left(d_{j}\right)$. As restrições $(7)$ são de domínio das variáveis de decisão. 


\section{Resultados Computacionais}

Um conjunto de 810 instâncias geradas aleatoriamente foi utilizado a fim de explorar o desempenho e os limitantes de cada modelo de acordo com os parâmetros fornecidos. Todos os experimentos foram realizados em um computador com 16GB de memória RAM e processador i7, utilizando o CPLEX 12.8 para resolver o problema e linguagem OPL para codificar o modelo.

As 810 instâncias utilizadas foram as mesmas geradas em [5] com 30 produtos $(N J=30)$, objetos de comprimento $1200(L=1200)$ e com 20, 30 e 40 tamanhos de itens diferentes $(N I)$. O número de espessura das matérias-primas $(N K)$ possui 2 cenários: 2 ou 8 . O número de modos alternativos por produto $(N M)$ tem três opções: 5,10 ou 15. Os tamanhos de itens $\left(l_{i}\right)$ variam em: pequenos $\left(l_{i} \in U[120,360]\right) ;$ grandes $\left(l_{i} \in U[360,840]\right)$; variados $\left(l_{i} \in U[120,840]\right)$. Por fim, o custo das matérias-primas tem três alternativas: idênticos $\left(\theta_{k}=1\right)$; homogêneos $\left(\theta_{k} \in U[1,10]\right)$; heterogêneos $\left(\theta_{k} \in U[1,100]\right)$. A notação $U$ representa a distribuição uniforme em um conjunto discreto.

As demandas dos produtos finais foram geradas sempre entre 1 e 10 unidades $\left(d_{j} \in U[1,10]\right)$. As quantidades exigidas de um item $i$ de determinada espessura $k$ no modo alternativo $m\left(b_{i k m}\right)$ também foram geradas aleatoriamente, considerando $98 \%$ de chances de ser nulo $\left(b_{i k m}=0\right)$ e $2 \%$ seguindo uma distribuição uniforme entre 2 e 8 unidades $\left(b_{i k m} \in U[2,8]\right)$. O limite de $98 \%$ foi inspirado no exemplo industrial de [5], onde $98 \%$ dos elementos $b_{i k m}$ eram iguais a 0 . A combinação desses cenários totalizam 162 conjuntos de parâmetros, para os quais 5 instâncias diferentes foram geradas aleatoriamente, totalizando 810 instâncias.

Tabela 1: Comparação dos limitantes superiores de FA em relação à GC (em \%).

\begin{tabular}{|c|c|c|c|c|c|c|c|c|c|c|c|c|}
\hline \multirow{2}{*}{ NK } & \multirow{2}{*}{ NM } & \multirow{2}{*}{ NI } & \multicolumn{3}{|c|}{$l_{i}$ (pequenos) } & \multicolumn{3}{|c|}{$l_{i}($ variados $)$} & \multicolumn{3}{|c|}{$l_{i}$ (grandes) } & \multirow{2}{*}{ Média } \\
\hline & & & $\theta_{h t}{ }^{1}$ & $\theta_{h m}{ }^{2}$ & $\theta_{i d}{ }^{3}$ & $\theta_{h t}$ & $\theta_{h t}$ & $\theta_{i d}$ & $\theta_{h t}$ & $\theta_{h m}$ & $\theta_{i d}$ & \\
\hline \multirow[t]{9}{*}{2} & 5 & 20 & 0,38 & 0,00 & 0,25 & 0,02 & 0,00 & 0,25 & 0,02 & 0,05 & 0,10 & 0,12 \\
\hline & & 30 & 0,17 & $-0,09$ & 0,00 & 0,06 & 0,02 & 0,00 & 0,13 & 0,00 & 0,00 & 0,03 \\
\hline & & 40 & 0,09 & $-0,02$ & 0,00 & 0,06 & 0,09 & 0,00 & 0,15 & 0,06 & 0,07 & 0,06 \\
\hline & 10 & 20 & 0,23 & 0,21 & 0,00 & 0,04 & 0,25 & 0,00 & 0,11 & 0,03 & 0,00 & 0,10 \\
\hline & & 30 & 0,04 & 0,08 & 0,27 & 0,43 & 0,12 & 0,00 & 0,02 & 0,15 & 0,18 & 0,14 \\
\hline & & 40 & 0,17 & $-0,04$ & 0,00 & $-0,02$ & 0,14 & 0,00 & 0,10 & 0,03 & 0,44 & 0,09 \\
\hline & 15 & 20 & 0,19 & 0,00 & 0,00 & 0,00 & 0,19 & 0,18 & 0,00 & 0,00 & 0,00 & 0,06 \\
\hline & & 30 & $-0,11$ & $-0,09$ & 0,00 & 0,38 & 0,00 & 0,22 & 0,07 & 0,11 & 0,10 & 0,08 \\
\hline & & 40 & $-0,12$ & $-0,53$ & 0,32 & 0,06 & 0,18 & 0,00 & 0,11 & 0,02 & 0,00 & 0,00 \\
\hline \multirow[t]{9}{*}{8} & 5 & 20 & 0,09 & 0,09 & $-0,16$ & 0,05 & 0,06 & 0,00 & 0,03 & 0,01 & 0,09 & 0,03 \\
\hline & & 30 & 0,02 & $-0,21$ & $-0,15$ & 0,08 & $-0,02$ & $-0,03$ & 0,02 & 0,09 & 0,00 & $-0,02$ \\
\hline & & 40 & $-0,66$ & $-0,18$ & $-0,22$ & 0,04 & 0,03 & 0,02 & 0,00 & 0,03 & 0,01 & $-0,10$ \\
\hline & 10 & 20 & $-0,04$ & $-0,34$ & $-0,15$ & 0,24 & 0,07 & 0,06 & 0,09 & 0,06 & 0,00 & 0,00 \\
\hline & & 30 & $-0,12$ & $-0,18$ & $-0,06$ & 0,00 & $-0,02$ & 0,00 & 0,02 & 0,11 & 0,03 & $-0,02$ \\
\hline & & 40 & $-0,33$ & $-0,27$ & $-0,47$ & 0,01 & $-0,02$ & $-0,03$ & 0,06 & 0,01 & 0,04 & $-0,11$ \\
\hline & 15 & 20 & $-0,30$ & 0,44 & $-0,32$ & 0,29 & 0,11 & 0,14 & 0,03 & 0,05 & 0,06 & 0,06 \\
\hline & & 30 & $-0,26$ & $-0,25$ & 0,25 & 0,00 & 0,10 & 0,02 & 0,02 & 0,00 & 0,12 & 0,00 \\
\hline & & 40 & $-0,24$ & $-0,37$ & $-0,18$ & $-0,03$ & $-0,02$ & 0,07 & 0,04 & 0,03 & 0,04 & $-0,07$ \\
\hline & Média & & $-0,04$ & $-0,10$ & $-0,03$ & 0,10 & 0,07 & 0,05 & 0,06 & 0,05 & 0,07 & 0,02 \\
\hline
\end{tabular}


Tabela 2: Comparação dos limitantes inferiores de FA em relação à GC (em \%).

\begin{tabular}{|c|c|c|c|c|c|c|c|c|c|c|c|c|}
\hline \multirow{2}{*}{ NK } & \multirow{2}{*}{ NM } & \multirow{2}{*}{ NI } & \multicolumn{3}{|c|}{$l_{i}$ (pequenos) } & \multicolumn{3}{|c|}{$l_{i}$ (variados) } & \multicolumn{3}{|c|}{$l_{i}$ (grandes) } & \multirow{2}{*}{ Média } \\
\hline & & & $\theta_{h t}$ & $\theta_{h m}$ & $\theta_{i d}$ & $\theta_{h t}$ & $\theta_{h t}$ & $\theta_{i d}$ & $\theta_{h t}$ & $\theta_{h m}$ & $\theta_{i d}$ & \\
\hline \multirow[t]{9}{*}{2} & \multirow[t]{3}{*}{5} & 20 & 0,13 & 0,26 & 0,59 & 0,20 & 0,21 & 0,16 & 0,11 & 0,17 & 0,09 & 0,21 \\
\hline & & 30 & 0,20 & 0,28 & 0,56 & 0,09 & 0,08 & 0,23 & 0,15 & 0,07 & 0,19 & 0,21 \\
\hline & & 40 & 0,08 & 0,17 & 0,44 & 0,03 & 0,11 & 0,34 & 0,08 & 0,04 & 0,16 & 0,16 \\
\hline & \multirow{3}{*}{10} & 20 & 0,23 & 0,29 & 0,87 & 0,09 & 0,45 & 0,50 & 0,09 & 0,09 & 0,17 & 0,31 \\
\hline & & 30 & 1,05 & 0,32 & 0,62 & 0,17 & 0,23 & 0,55 & 0,05 & 0,18 & 0,17 & 0,37 \\
\hline & & 40 & 0,03 & 0,19 & 0,75 & 0,03 & 0,26 & 0,19 & 0,19 & 0,13 & 0,23 & 0,22 \\
\hline & \multirow[t]{3}{*}{15} & 20 & 0,31 & 0,60 & 0,70 & 0,22 & 0,19 & 0,47 & 0,10 & 0,11 & 0,31 & 0,33 \\
\hline & & 30 & 0,02 & 0,10 & 0,85 & 0,13 & 0,32 & 0,48 & 0,09 & 0,22 & 0,21 & 0,27 \\
\hline & & 40 & 0,12 & 0,01 & 0,61 & 0,11 & 0,18 & 0,44 & 0,08 & 0,08 & 0,34 & 0,22 \\
\hline \multirow[t]{9}{*}{8} & \multirow[t]{3}{*}{5} & 20 & 0,08 & 0,08 & 0,05 & 0,23 & 0,23 & 0,10 & 0,11 & 0,14 & 0,19 & 0,14 \\
\hline & & 30 & 0,01 & 0,02 & 0,00 & 0,07 & 0,08 & 0,08 & 0,10 & 0,11 & 0,10 & 0,06 \\
\hline & & 40 & 0,00 & 0,00 & 0,00 & 0,04 & 0,02 & 0,02 & 0,10 & 0,08 & 0,07 & 0,04 \\
\hline & \multirow[t]{3}{*}{10} & 20 & 0,16 & 0,08 & 0,09 & 0,22 & 0,31 & 0,26 & 0,37 & 0,25 & 0,36 & 0,23 \\
\hline & & 30 & 0,02 & 0,03 & 0,01 & 0,10 & 0,06 & 0,08 & 0,27 & 0,23 & 0,27 & 0,12 \\
\hline & & 40 & 0,00 & 0,01 & 0,00 & 0,06 & 0,06 & 0,04 & 0,09 & 0,11 & 0,13 & 0,06 \\
\hline & \multirow[t]{3}{*}{15} & 20 & 0,16 & 0,08 & 0,23 & 0,38 & 0,53 & 0,28 & 0,34 & 0,19 & 0,41 & 0,29 \\
\hline & & 30 & 0,07 & 0,01 & 0,01 & 0,20 & 0,14 & 0,04 & 0,20 & 0,11 & 0,18 & 0,11 \\
\hline & & 40 & 0,01 & 0,00 & 0,01 & 0,05 & 0,02 & 0,06 & 0,14 & 0,15 & 0,12 & 0,06 \\
\hline & édia & & 0,15 & 0,14 & 0,36 & 0,14 & 0,19 & 0,24 & 0,15 & 0,14 & 0,21 & 0,19 \\
\hline
\end{tabular}

Tabela 3: Comparação dos tempos de solução de FA em relação à GC (em \%).

\begin{tabular}{|c|c|c|c|c|c|c|c|c|c|c|c|c|}
\hline \multirow{2}{*}{ NK } & \multirow{2}{*}{ NM } & \multirow{2}{*}{ NI } & \multicolumn{3}{|c|}{$l_{i}($ pequenos $)$} & \multicolumn{3}{|c|}{$l_{i}(\operatorname{variados})$} & \multicolumn{3}{|c|}{$l_{i}$ (grandes) } & \multirow{2}{*}{ Média } \\
\hline & & & $\theta_{h t}$ & $\theta_{h m}$ & $\theta_{i d}$ & $\theta_{h t}$ & $\theta_{h t}$ & $\theta_{k}$ & $\theta_{h t}$ & $\theta_{h m}$ & $\theta_{i d}$ & \\
\hline \multirow[t]{9}{*}{2} & \multirow[t]{3}{*}{5} & 20 & $-88,8$ & $-6,5$ & 93,5 & $-187,8$ & $-85,3$ & $-75,7$ & 9,5 & 62,6 & 98,2 & $-20,0$ \\
\hline & & 30 & 12,1 & $-20,6$ & 23,6 & $-113,3$ & $-62,5$ & 81,2 & $-364,8$ & 94,0 & 98,6 & $-28,0$ \\
\hline & & 40 & 42,6 & $-8,2$ & 94,8 & $-7,5$ & $-44,1$ & 97,0 & $-118,2$ & 98,5 & 98,2 & 28,1 \\
\hline & \multirow[t]{3}{*}{10} & 20 & 39,9 & 61,5 & 94,7 & $-71,2$ & 95,5 & 97,4 & 53,6 & $-256,5$ & 99,1 & 23,8 \\
\hline & & 30 & 10,8 & $-22,0$ & 86,1 & $-131,2$ & 47,3 & 94,7 & 91,1 & $-21,5$ & 99,0 & 28,3 \\
\hline & & 40 & 3,0 & $-24,2$ & 29,0 & $-17,3$ & 58,5 & 6,5 & 62,3 & 95,6 & 99,2 & 34,7 \\
\hline & \multirow[t]{3}{*}{15} & 20 & 78,6 & $-26,5$ & 43,8 & $-125,3$ & 71,5 & 97,8 & 80,7 & 98,8 & 98,8 & 46,5 \\
\hline & & 30 & $-30,3$ & $-144,6$ & 91,9 & 44,4 & 89,2 & 93,9 & $-188,3$ & 89,4 & 96,4 & 15,8 \\
\hline & & 40 & 48,8 & 16,2 & 76,7 & $-18,7$ & 63,9 & 89,8 & $-16,2$ & 42,3 & 99,1 & 44,7 \\
\hline \multirow[t]{9}{*}{8} & \multirow[t]{3}{*}{5} & 20 & 58,4 & 61,6 & 55,8 & 9,4 & 43,6 & $-7,7$ & 82,7 & $-66,7$ & 91,3 & 36,5 \\
\hline & & 30 & 80,3 & 83,1 & 77,9 & 55,4 & 30,1 & 57,9 & $-3,2$ & 36,9 & 31,6 & 50,0 \\
\hline & & 40 & 89,4 & 71,0 & 56,0 & 78,0 & 81,8 & 76,3 & 64,9 & 73,4 & 75,5 & 74,0 \\
\hline & \multirow[t]{3}{*}{10} & 20 & 75,7 & 71,5 & 60,6 & 7,4 & $-65,0$ & $-8,9$ & 88,5 & 46,6 & 97,9 & 41,6 \\
\hline & & 30 & 79,7 & 81,2 & 83,2 & 69,9 & 77,7 & 52,6 & $-70,7$ & 86,6 & 98,5 & 62,1 \\
\hline & & 40 & 92,9 & 91,6 & 89,5 & 74,2 & 87,0 & 75,7 & 58,1 & 58,7 & 99,0 & 80,7 \\
\hline & \multirow[t]{3}{*}{15} & 20 & 56,5 & 62,6 & 52,5 & 5,8 & 20,6 & $-23,6$ & $-32,6$ & $-34,5$ & 96,9 & 22,7 \\
\hline & & 30 & 83,6 & 83,1 & 83,4 & 56,9 & 42,9 & 71,3 & 93,8 & 55,1 & 98,7 & 74,3 \\
\hline & & 40 & 90,9 & 91,4 & 89,8 & 76,1 & 80,4 & 79,1 & 46,6 & 42,8 & 77,1 & 74,9 \\
\hline \multicolumn{3}{|c|}{ Média } & 45,8 & 29,0 & 71,3 & $-10,8$ & 35,2 & 53,1 & $-3,5$ & 33,5 & 91,8 & 38,4 \\
\hline
\end{tabular}


A formulação de fluxo em arcos proposta neste trabalho será chamada de FA. No que se refere aos critérios de redução de arcos utilizados por [9], o modelo implementado neste artigo utilizou o critério de ordenação decrescente dos itens, e de eliminação dos arcos de perda. O critério relacionado à demanda, para o caso do problema aqui mostrado, não influenciou os resultados do modelo e aumentou o tempo para a geração da rede. Será chamado de GC o método de solução proposto em [5], que é baseado na formulação proposta em [4] e numa heurística para obtenção de uma solução inteira.

Os dados foram submetidos ao modelo e o tempo limite de execução para encontrar a solução inteira foi limitado a 60 segundos, sendo o mesmo tempo utilizado para encontrar a solução inteira depois da geração de colunas na formulação de [5]. Contudo, dessas 810 instâncias, 9 não obtiveram solução viável nos 60 segundos. Para essas instâncias, aumentou-se o tempo gradativamente até alcançar uma solução.

Os limitantes superiores, inferiores e o tempo de solução do modelo FA são comparados com os do modelo GC. A comparação é feita usando variação percentual. A equação (8) foi utilizada para calcular a variação percentual dos limitantes superiores e dos tempos de solução entre as duas formulações para cada instância $i$. Já a equação (9) foi utilizada para o cálculo da variação dos limitantes inferiores entre os dois modelos. Dessa forma, as Tabelas 1, 2 e 3 apresentam a comparação dessas características para um dado conjunto de parâmetros. Valores positivos indicam a porcentagem que os valores de FA melhoraram em relação a GC. Caso o valor seja negativo, isso significa que os atributos em FA pioraram em relação ao modelo GC.

$$
\begin{aligned}
\text { variação percentual }_{i} & =1-\frac{F A_{i}}{G C_{i}} \\
\text { variação percentual }_{i} & =\frac{F A_{i}}{G C_{i}}-1
\end{aligned}
$$

As soluções encontradas pelo modelo de fluxo em arco melhoraram, em média, $0,02 \%$ em relação ao modelo de geração de colunas. Na maioria das vezes (cerca de $56 \%$ das instâncias), as soluções foram as mesmas para ambas as formulações. Na Tabela 1, é possível observar que o modelo GC é superior, principalmente, em instâncias de itens pequenos e maior NK.

Em relação aos limitantes inferiores, a Tabela 2 indica que, para todos os conjuntos de dados, a formulação de fluxo em arcos possui limitantes inferiores maiores ou iguais aos limitantes encontrados na formulação GC. Esse valor é, em média, 0,19\% maior. Em geral, os limitantes inferiores do modelo de fluxo em arcos apresentaram maior vantagem nas instâncias com menores quantidades de espessura das barras.

Em $86 \%$ das instâncias, o modelo de fluxo em arcos encontrou uma solução em um tempo menor. Conforme a Tabela 3, a média dos tempos de solução foi $38,4 \%$ menor para o modelo de fluxo em arcos comparado ao modelo da literatura. Ainda é possível notar que, nos itens com maiores quantidades de matérias-primas, o impacto da formulação no tempo de resolução é maior, enquanto que, para instâncias com menores quantidades de espessuras de matérias-primas, a formulação, por vezes, precisou de mais tempo para encontrar a solução.

\section{Conclusão}

Neste artigo são realizados estudos de desempenho para uma nova formulação do Problema de Corte de Estoque com modos alternativos de manufatura (PCEMA-1D) encontrado na literatura. Além de fornecer um modelo alternativo baseado na formulação de fluxo de arcos, esse artigo também comparou essa nova formulação com os resultados de [5], que primeiro introduziu a integração do problema de corte de estoque com diferentes modos de manufatura desenvolvendo um método de solução baseado no procedimento de geração de colunas. 
Um total de 810 instâncias aleatórias foram utilizadas a fim de testar as influências dos parâmetros e comparar a formulação com o modelo da literatura. Os resultados apontam que o modelo de fluxo em arcos consegue obter soluções com redução de 38,4\% no tempo, em média, apesar de não apresentar melhoria significativa nessas soluções. Além disso, o modelo de fluxo em arcos alcança maiores limitantes inferiores.

Visto que em 9 instâncias o modelo de fluxo em arcos não conseguiu encontrar soluções no tempo determinado, em trabalhos futuros estudaremos o modelo reflect proposto por [2], que reduz ainda mais o número de variáveis e restrições do modelo. Novos estudos também englobam a extensão do problema para o caso multi-período, onde há a possibilidade de estocar itens e produtos finais.

\section{Referências}

[1] Delorme, M., Iori, M. e Martello, S. Bin packing and cutting stock problems: Mathematical models and exact algorithms, European Journal of Operational Research, 255:1-20, 2016. DOI: 10.1016/j.ejor.2016.04.030.

[2] Delorme, M. e Iori, M. Enhanced pseudo-polynomial formulations for bin packing and cutting stock problems, INFORMS Journal on Computing, 32:101-119, 2020. DOI: 10.1287/ijoc.2018.0880.

[3] Dyckhoff, H. A typology of cutting and packing problems, European Journal of Operational Research, 44:145-159, 1990. DOI: 10.1016/0377-2217(90)90350-K.

[4] Gilmore, P. C. e Gomory, R. E. A linear programming approach to the cutting-stock problem, Operations Research, 9:849-859, 1961. DOI: 10.1287/opre.9.6.849.

[5] Lemos, F. K., Cherri, A. e De Araujo, S. The cutting stock problem with multiple manufacturing modes applied to a construction industry. International Journal of Production Research, 1-19, 2020. DOI: 10.1080/00207543.2020.1720923.

[6] Liu, Q., Geng, X., Dong, M., Lv, W. e Ye, C. Scheduling optimization of design stream line for production research and development projects, Engineering Optimization, 49:896-914, 2017. DOI: 10.1080/0305215X.2016.1216113.

[7] Melega, G. M., de Araujo, S. A. e Morabito, R. Mathematical model and solution approaches for integrated lot-sizing, scheduling and cutting stock problems, Annals of Operations Research, 295:695-736, 2020. DOI: 10.1007/s10479-020-03764-9.

[8] Santoro, M. C. e Lemos, F. K. Irregular packing: Milp model based on apolygonal enclosure, Annals of Operations Research, 235:693-707, 2015. DOI: 10.1007/s10479-015-1971-9.

[9] Valério De Carvalho, J. Lp models for bin packing and cutting stock problems, European Journal of Operational Research, 141:253-273, 2002. DOI: 10.1016/S0377-2217(02)00124-8.

[10] Wäscher, G., Haußner, H. e Schumann, H. An improved typology of cutting and packing problems, European Journal of Operational Research, 183:1109-1130, 2007. DOI: 10.1016/j.ejor.2005.12.047.

[11] Wolsey, L. A. Valid inequalities, covering problems and discrete dynamic programs, Annals of Discrete Mathematics, 1:527-538, 1977. DOI: 10.1016/S0167-5060(08)70758-1.

[12] Wuttke, D. A. e Heese, H. S. Two-dimensional cutting stock problem with sequence dependent setup times, European Journal of Operational Research, 265:303-315, 2018. DOI: 10.1016/j.ejor.2017.07.036. 